\title{
A generalized 3D inverted pendulum model to represent human normal walking
}

\author{
Sophie Sakka \\ IRCCyN, Robotics Dpt., Nantes \\ University of Poitiers, France \\ Email: Sophie.Sakka@irccyn.ec-nantes.fr
}

\author{
Chris Hayot and Patrick Lacouture \\ Pprime Research Institute, ROBIOSS Dpt. \\ University of Poitiers, France \\ Email: \{hayot,lacouture\}@univ-poitiers.fr
}

\begin{abstract}
This paper compares different inverted pendulum models to represent the stance phase of human normal walking. We have developed a model which takes into account the mechanism of the foot during the single support phase, by defining a pivot point under the ground level. Similarly to other models, the pivot point as well as the rod length remain constant during the complete single support phase. Lowering the position of the pivot point allows reducing the vertical amplitude of the center of mass (CoM) trajectory and therefore approaching the real CoM trajectory. Another big advantage of this model is that it can be easily adapted to pathological walking as the walking symmetry hypothesis -needed to build classical inverted pendulum models, is not used. We have measured the whole body kinematics of 5 healthy male subjects and set a reference CoM trajectory based on multibody modeling of the human body (16 segments). Then, we have determined a common mathematical definition of three inverted pendulum models: the classical IP-3D, the EIP-3D and our GIP-3D model. To insure continuity of the approached trajectories, a double support phase is represented by a simple pendulum which is a homothetic transformation of the inverted pendulum defining the previous single support phase, except for our GIP-3D model which may include asymmetric walking parameters.
\end{abstract}

\section{INTRODUCTION}

The calculation of the mechanical energy used for normal walking is based on the 3D trajectory and velocity characteristics of the center of mass (CoM) displacements. It looked then natural to propose evaluation models of normal walking focusing on the estimation of these trajectory and velocity characteristics, avoiding the use of heavy experimental setting needed by a multibody modeling of the human body. Two main approaches emerged: the inverted pendulum models and the major determinants of walking.

Indeed, locomotion can be approximate from the external forces point of view as the displacement of the center of mass $(\mathrm{CoM})$ in 3D space. During normal walking, the CoM trajectory follows a cyclic sinusoidal pattern, and it seemed natural representing it with pendulum models: an inverted pendulum for the single support phases of the walking cycle, and a simple pendulum for the double support ones [4], [15]. When not pathological, walking is assumed perfectly regular and symmetric: one is able to predict the CoM trajectory knowing the characteristics of the subject anatomy and gait comfortable speed. Then the approximation of the CoM trajectory is available for healthy subjects walking at different speeds using a pendulum model from the spatial and temporal gait parameters [5], [8], [11].

In this paper, we focused on the pendulum representation of normal walking. Our main motivation was to improve the existing models by getting closer to a more realistic 3D trajectory of the CoM while reducing the heaviness of the experimental setting needed by multibody models. Studying the dynamics experimental data: the reference CoM (calculated using multibody modeling) and the CoP (measured using force plates) trajectories during the swing phase of normal walking, we have observed a convergence of the straight lines joining these two points. We could then extract a pivot point for the pendulum representation based on a real mechanism of normal walking. Still, its position is not on the ground level as assumed by all the existing pendulum approaches, but about $38 \mathrm{~cm}$ below. This situation was already suggested by some authors, assuming that if the motion of the center of pressure is taken into account, it is equivalent to considering the pivot point of walking under the ground level [1], [2], [9]. To our knowledge, no further studies were realized to actually put this assessment in evidence. We have set a three dimensional pendulum model based on the observed pivot point and compared our results to the reference CoM trajectory and two approximate CoM trajectories using Zijlstra's IP-3D and Hayot's EIP3D models. We first define the three pendulum models in section 2 using a common mathematical representation based on homogeneous transformation matrices. Then we expose our results in section 3 and discuss them in section 4 .

\section{PRotocol AND POST-TREATMENT OF THE DATA}

\section{A. Subjects, materials and experimental protocol}

The experimentations were realized with 5 healthy male subjects aged $\mathrm{XX} \pm \mathrm{XX}$ who gave their oral agreement to participate in this study. Their instructions consisted in walking at their comfortable speed $(1,30 \pm 0,22 \mathrm{~m} / \mathrm{s})$ following a 10-meter walk way. Average step length was $1,322 \pm 0,041 \mathrm{~m}$.

Each subject was equipped with 58 markers and performed three walking trials. $3 \mathrm{~d}$ trajectories of the markers were captured at the frequency of $100 \mathrm{~Hz}$ using an optical motion capture system (Vicon, Oxford, UK). The ground reaction force and momentum were registered using two $3 \mathrm{~d}$ force 
plates (Kistler 5233A and 9287B, Winterthur, Switzerland) at the frequency $900 \mathrm{~Hz}$. The efforts data were synchronized with the kinematics data and the measurements were realized for a complete walking cycle.

\section{B. Use and post treatment of the data}

The human body was modeled by 16 rigid segments representing the feet, legs, thighs, hands, forearms, arms, pelvis, abdomen, thorax and head (including neck). Local frames were defined for each segment in agreement with the recommendations of the International Society of Biomechanics [12], [13], and bodies masses, CoM trajectory and moment of inertia were calculated using predictive equations and data from Zatsiorsky [14]. the reference kinematics of the movement could be calculated based on the homogeneous matrix formulation of the equations of motion [7]. The reference trajectory of the CoM was obtained from the weighted mean of the bodies CoM trajectories and the respective masses:

$$
\left[\begin{array}{c}
\mathbf{O C} \\
1
\end{array}\right]_{\mathcal{R}_{0}}=\frac{1}{M} \sum_{i=1}^{16} m_{i} T_{0}^{i}\left[\begin{array}{c}
\mathbf{O}_{\mathbf{i}} \mathbf{C}_{\mathbf{i}} \\
1
\end{array}\right]_{\mathcal{R}_{i}}
$$

OC denotes the absolute position of the CoM, $m_{i}$ is the mass of the $i^{t h}$ body and $M=\sum_{i=1}^{16} m_{i}$ is the total mass of the subject. $T_{j}^{k}$ denotes the homogeneous transformation matrix from $\mathcal{R}_{j}$ to $\mathcal{R}_{k}, \mathcal{R}_{0}$ being the reference frame.

Force and torque data were used to determine the CoP trajectory as well as to detect temporal characteristics of the walking cycle: contact instants at heal strike of the leading (LHS) and trailing (THS) limb, and at toe off (LTO and LTO, respectively).

The data extracted from all the trials were averaged after normalization with time and the reference static CoM height.

\section{Pendulum MOdels OF HUMAN WALKING}

\section{A. Pendulum models}

Inverted pendulum models usually describe the single support phase of normal walking under the assumption that

- The gait is in steady state: there is no variation of CoM walking speed or trajectory amplitude during observation;

- The walking is symmetric: similar motion is performed by the left and the right leg;

- The system is conservative; Painlevé first integral is valid (conservation of the mechanical energy during walking)

The double support phase is rarely represented, or more precisely is widely approached either by a straight line linking two single support phases in the case of the inverted pendulum representation [6], [15], or just ignored if using the compass gait representation [10]. A direct consequence is that the CoM velocity is not continuous at the transition instants between single and double support phases. In 1997, Zijlstra et al. [15] introduced a continuous representation

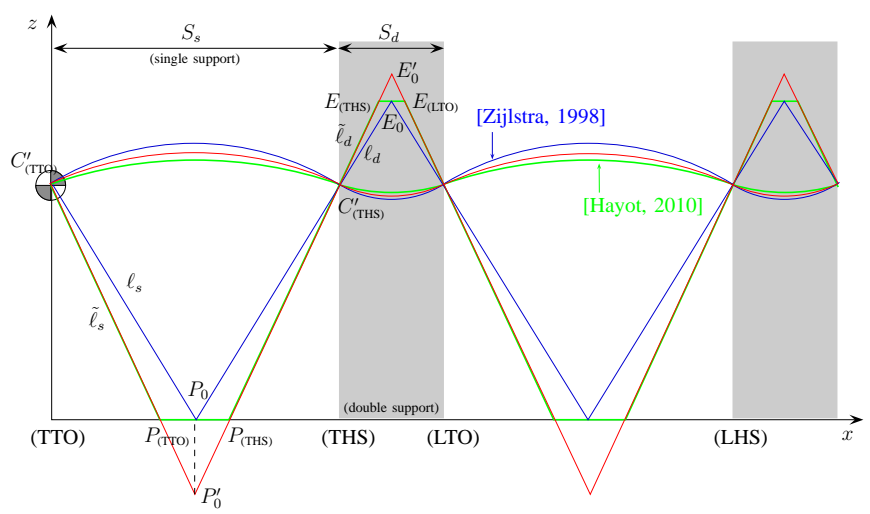

Fig. 1. Pendulum representation of normal walking, projection in the sagittal plane. The amplitude of the CoM trajectory is overestimated using [15]'s approach, underestimated using [4]'s approach.

of the CoM trajectory assimilating its oscillations in steady state to the ones of a vibratory system. In this model, the amplitude of the vibrations relies on the walking subject's morphological characteristics and the frequency is set according to the walking speed and the length of the steps. The pivot point of the pendulum, $P_{0}$, is set in the middle of the trajectory of the center of pressure $(\mathrm{CoP})$ under the foot as illustrated by the blue lines in Fig. 1. This model insures the continuity of the CoM trajectory over the gait cycle, but shows the main disadvantage to widely overestimate the amplitude of its trajectory. Dealing with this major drawback and under the assumption that the determinants of walking had to be taken into account in a realistic pendulum representation of normal walking, Hayot et al. [4] extended the model by introducing the mechanism of the foot during single support phase. The resulting model is represented in black lines in Fig. 1: similarly to Zijlstra, the length of the pendulum rod is measured at TTO (trailing toes off) instant and remains constant all over the single support phase, but the pivot point on the ground moves during the phase and is superposed with the CoP at each instant. Hayot et al. showed that the amplitude of the CoM trajectory got closer to the actual one by comparing their model with a reference trajectory extracted from multibody modeling of the tested subjects. Nevertheless, the approached trajectory underestimates, this time, the trajectory amplitude. Another difficulty of using this model is the motion of the pivot point on the ground, which requires the computation of the position of the CoP under the foot at each instant of the single support phase.

Both Zijlstra's and Hayot's models rebuilt the double support phase using an homothetic transformation of the single support phase. The center of the homothety is the position of the approached CoM $\left(C^{\prime}\right)$ at the transition instants between a single support phase and the next double support phase (THS: Trailing Heal Strike). The homothety ratio $k_{0}$ is the quotient of the distance performed by the CoM during double support phase $\left(S_{d}\right)$ over the distance performed during the 
single support phase $\left(S_{s}\right)$ (Fig. 1):

$$
k_{0}=\frac{S_{d}}{S_{s}} .
$$

Therefore knowing the characteristics of the inverted pendulum at TTO (the positions of the $\mathrm{CoM}$ and $\mathrm{CoP}$ ) allows completely rebuilding the normal gait cycle of the subject.
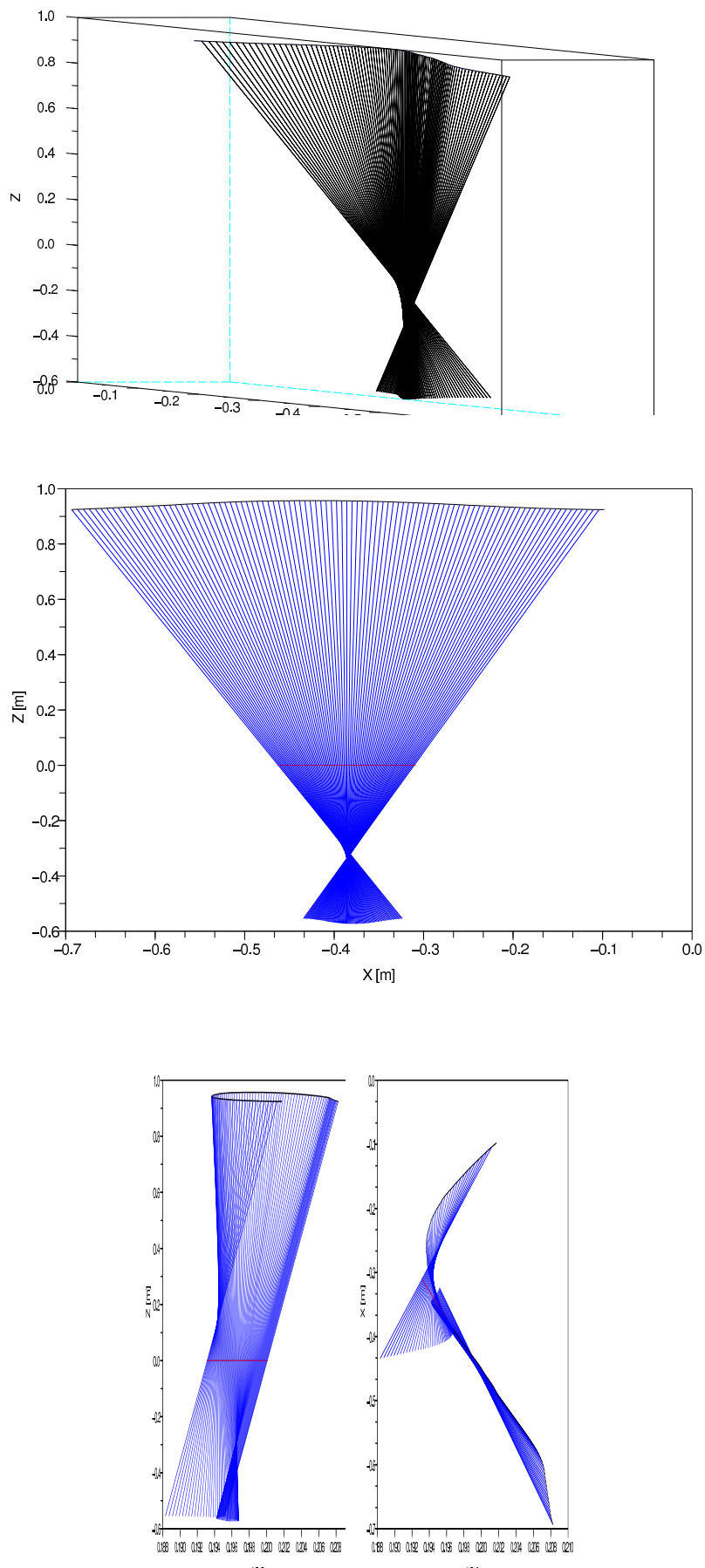

㭣

Fig. 2. Views of the support lines (joining the CoM and the CoP) at each time step of the single support phase. The black line represents the trajectory of the CoM and the red line the trajectory of the CoP.
Figure 2 shows the three dimensional evolution with time of the segment lines $[P C]$ during single support, and the three associated views in the $\mathrm{X}-\mathrm{Z}$ (sagittal view), Y-Z (frontal view) and $X-Y$ (top or horizontal view) planes. In this figure, the reference $\mathrm{CoM}$ and $\mathrm{CoP}$ trajectories are also represented. These segments are called support lines in what follows, as they join the two points characterizing the dynamics of the walking motion (the $\mathrm{CoM}$ and the $\mathrm{CoP}$ ) from a global point of view. The extension of these support lines in space show that they are converging in an area located around mid stance and 34 centimeters under the ground level. Using this observation, we have defined a generalized pendulum model represented in the sagittal plane in Fig. 1 using red lines. Observing the three projections in the X-Y, Y-Z and $\mathrm{X}-\mathrm{Z}$ planes, it is very difficult to extract a regular pattern of the pivot area which would allow determining a single pivot point for our inverted pendulum representation. We will then focus on this particular area in order to precisely define the pivot point $P_{0}^{\prime}$ represented in Fig 1.

\section{B. Mathematical formulation}

Let $\mathcal{R}_{0}=\left(O, \mathbf{x}_{\mathbf{0}}, \mathbf{y}_{\mathbf{0}}, \mathbf{z}_{\mathbf{0}}\right)$ be the reference frame, $\mathcal{R}_{P_{k}}=$ $\left(P_{k}, \mathbf{x}_{\mathbf{P}_{\mathbf{k}}}, \mathbf{y}_{\mathbf{P}_{\mathbf{k}}}, \mathbf{z}_{\mathbf{P}_{\mathbf{k}}}\right)$ the frame attached to the considered inverted pendulum model during single support and $\mathcal{R}_{E_{k}}=$ $\left(E_{k}, \mathbf{x}_{\mathbf{E}_{\mathbf{k}}}, \mathbf{y}_{\mathbf{E}_{\mathbf{k}}}, \mathbf{z}_{\mathbf{E}_{\mathbf{k}}}\right)$ the frame attached to the linear pendulum during the double support phase. Here, $P_{k}$ (respectively $E_{k}$ ) may be replaced by the pivot point matching the considered model as summarized in Tab. I: either Zijlstra's continuous model (IP-3D): $P_{k}=P_{0}$ (respectively $E_{k}=E_{0}$ ), Hayot's extended model (EIP-3D): $P_{k}=P(t)$ (respectively $E_{k}=E(t)$ ) or our generalized model (GIP-3D): $P_{k}=P_{0}^{\prime}$ (respectively $E_{k}=E_{0}^{\prime}$ ) according to the notations described in Fig. 1. $C$ denotes the actual CoM of the observed subject, and $C^{\prime}$ is the approached CoM calculated by one of the pendulum models. At $t=\mathrm{TTO}, C^{\prime}$ and $C$ are considered identical which allows setting the length of the pendulum rod. Then the trajectory of $C^{\prime}$ in the reference frame is defined during the complete walking cycle as follows.

$$
\left[\begin{array}{c}
\mathbf{O C}^{\prime} \\
1
\end{array}\right]_{\mathcal{R}_{0}}=\left\{\begin{array}{cc}
T_{0}^{P_{k}}\left[\begin{array}{c}
\mathbf{P}_{\mathbf{k}} \mathbf{C}^{\prime} \\
1
\end{array}\right]_{\mathcal{R}_{P_{k}}} & t \in[T T O, T H S] \\
T_{0}^{E_{k}}\left[\begin{array}{c}
\mathbf{E}_{\mathbf{k}} \mathbf{C}^{\prime} \\
1
\end{array}\right]_{\mathcal{R}_{E_{k}}} & t \in[T H S, L T O]
\end{array}\right.
$$

The matrices $T_{0}^{i}$ are the homogeneous transformation matrices from the frame $\mathcal{R}_{i}$ to the reference frame $\mathcal{R}_{0}$.

$$
T_{0}^{i}=\left[\begin{array}{cccc}
\cos \alpha_{j} & \sin \alpha_{j} \sin \beta_{j} & \sin \alpha_{j} \cos \beta_{j} & x_{i} \\
0 & \cos \beta_{j} & -\sin \beta_{j} & y_{i} \\
-\sin \alpha_{j} & \cos \alpha_{j} \sin \beta_{j} & \cos \alpha_{j} \cos \beta_{j} & z_{i} \\
0 & 0 & 0 & 1
\end{array}\right]
$$

with $i=\left\{P_{k}, E_{k}\right\}$ and $j=\{s, d\}$. The indexes $s$ and $d$ refer to the single and double support phases, respectively. $\alpha(t)$ and $\beta(t)$ are the sweep angles, around the lateral and the frontal axis, respectively. The two vectors $\mathbf{P}_{\mathbf{k}} \mathbf{C}^{\prime}$ and $\mathbf{E}_{\mathbf{k}} \mathbf{C}^{\prime}$ 
are defined in their respective frames as follows.

$$
\mathbf{P}_{\mathbf{k}} \mathbf{C}^{\prime}=\ell_{s}^{k} \mathbf{z}_{P_{k}} \quad \mathbf{E}_{\mathbf{k}} \mathbf{C}^{\prime}=\ell_{d}^{k} \mathbf{z}_{E_{k}}
$$

where $\ell_{j}^{k}(j=\{s, d\})$ defines the rod lengths which remain constant over their respective support phases and are such that

1) $\ell_{s}^{k}$ denotes the length between the pivot point and the $\mathrm{CoM}$ at $t=\mathrm{TTO}$

2) at $t=$ THS, the three points $P_{k}, C^{\prime}$ and $E_{k}$ are aligned and

$$
\ell_{d}^{k}=k_{0} \ell_{s}^{k}
$$

TABLE I

3D PENDULUM MODELS CHARACTERISTICS DEFINING THE PIVOT POINTS AND THE ROD LENGTH FOR ZIJLSTRA'S CONTINUOUS MODEL (IP-3D), HAYOT'S EXTENDED MODEL TAKING THE MECHANISM OF THE FEET INTO ACCOUNT (EIP-3D) OR OUR GENERALIZED MODEL (GIP-3D).

\begin{tabular}{|c|c|c|c|c|}
\hline model $k$ & $P_{k}$ & $E_{k}$ & $\ell_{s}^{k}$ & $\ell_{d}^{k}$ \\
\hline IP-3D & $P_{0}$ & $E_{0}$ & $\ell_{s}$ & $\ell_{d}$ \\
\hline EIP-3D & $P(t)$ & $E(t)$ & $\ell_{s}$ & $\ell_{d}$ \\
\hline GIP-3D & $P_{0}^{\prime}$ & $E_{0}^{\prime}$ & $\ell_{s}$ & $\ell_{d}$ \\
\hline
\end{tabular}

Concerning the EIP-3D model, the horizontal variations of the pivot point $E$ during the double support phase are relative to the horizontal variations of the $\mathrm{CoP}$ during the single support phase:

$$
\delta(\mathbf{O E})=k_{0} \delta(\mathbf{O P})
$$

The three models we have described are 3D models and may represent the complete walking cycle of a subject by only measuring kinematics and dynamics of the normal walking during a single support phase. Still, the fact that the length of the pendulum rods remain constant all over the phases does not guaranty the continuity of the approached CoM trajectories at Leading Toe Off (LTO, Fig. 1) instant unless the measured support distance at TTO and LTO are exactly equal. In what follows, we will consider this hypothesis true.

\section{EXPERIMENTAL RESULTS}

We have compared the different inverted pendulum models to the reference in terms of CoM approached trajectories. For instance, Fig. 3 shows the average CoM trajectories in the sagittal, frontal and horizontal planes for the IP-3D, EIP-3D and GIP described in Fig. 1, as well as the reference CoM trajectory obtained using multibody representation of the human body (the black line). The main differences between these four cases are described in the following section.

To introduce the GIP approximation, we have used the observation that the support lines joining the CoM and the $\mathrm{CoP}$ were converging to a small area located $38 \mathrm{~cm}$ under the ground level, as shown in Fig. 2. We have considered
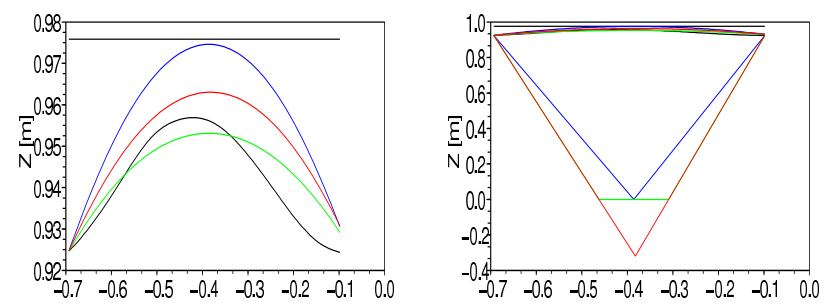

$X[\mathrm{~m}]$
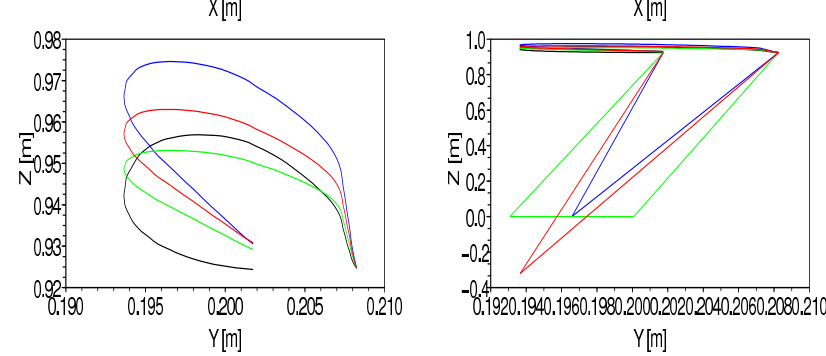

$\mathrm{Y}[\mathrm{m}]$

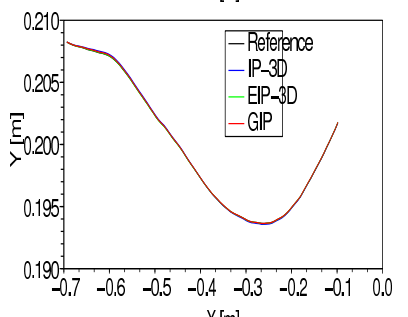

$X[\mathrm{~m}]$

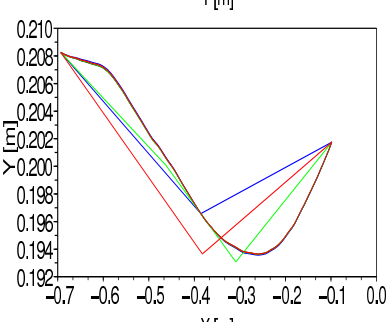

$X[\mathrm{~m}]$

Fig. 3. Reference trajectory of the CoM (black line) and its approximation using IP-3D (red line), EIP-3D (blue line) and GIP-3D (green line). The straight lines represent the respective pendulum rods.

that the pivot point of the GIP could be defined using the boundary support lines at TTO and THS and detecting the middle point of the shortest distance in space between these two lines. For this affirmation, we have closely observed the relative movements of the support lines with time during the complete single support phase, extracting the instantaneous rotation vector from one time step to the next one, which is illustrated in Fig. 6, as well as the minimal distances between the support lines (Fig. 4 and 5). We have calculated the weighted mean of the instantaneous pivot points which represent the crossing points between two support lines considered at two adjacent time steps. We have observed that it is very close to the GIP pivot point $P_{0}^{\prime}$ presented in section II-A (Fig. 1).

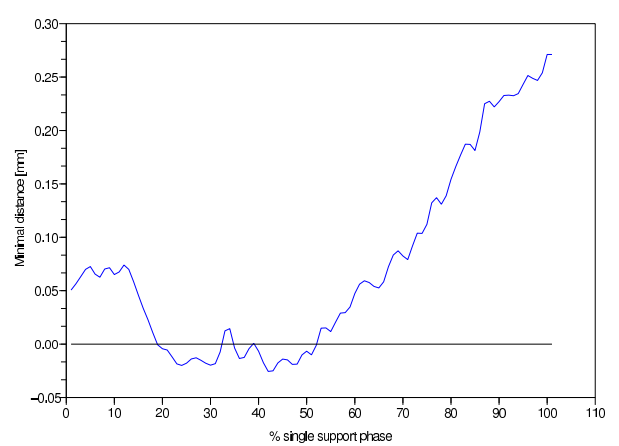

Fig. 4. The minimal distance between two adjacent support lines does not exceed $0.3 \mathrm{~mm}$. 

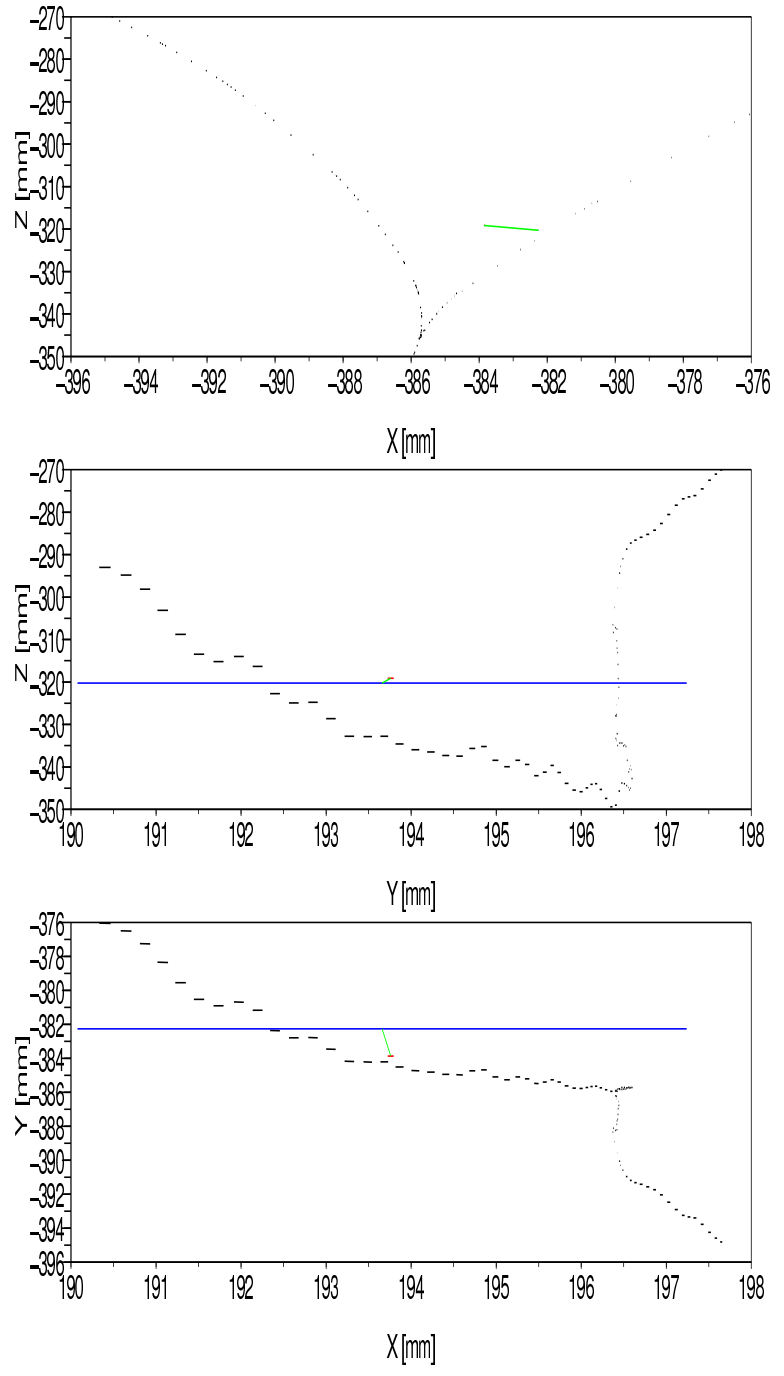

Fig. 5. Position of the pivot points with time. The black lines represent the minimal distance between two adjacent support lines at each time step; the red line is the weighted mean and the blue line is the minimal distance between the first (TTO) and last (THS) support line of the single support phase. The distance between the pivot points calculated using the weighted mean and the first and last support mean is represented by the green line.

\section{Discussion}

We have presented different inverted pendulum models adapted to normal walking under the hypotheses that walking is symmetrical and regular on flat floor. The classical continuous 3D inverted pendulum model (IP-3D), such as the one proposed by Zijlstra et al. [15], presents the main drawback to overestimate the amplitude of the CoM trajectory by representing it as an arc of circle which center is fixed on the ground level. An intermediary model between the inverted pendulum and the six determinants of gait representations of human walking was proposed by Hayot et al. [4]. The approached CoM trajectory gets closer to the real one as it is flattened compared to the IP-3D with the known consequences on energy and work estimations [6]. Still, this model slightly underestimate the CoM amplitude over the gait cycle [4]. Moreover, it is based on a moving pivot point which requires the measurement of
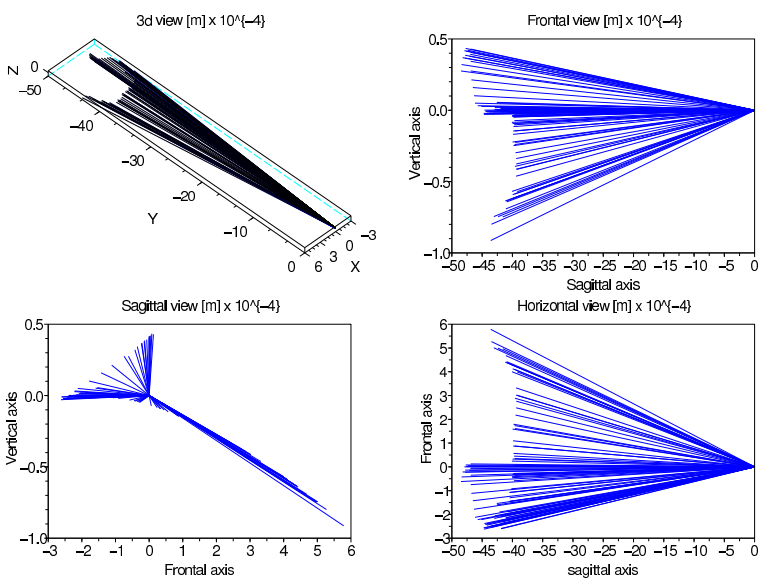

Fig. 6. Evolution of the instantaneous rotation vector of the support lines from a time step to the next one.

the CoP position at each time step. We have then proposed a generalized pendulum model after having observed that the support lines joining the $\mathrm{CoM}$ and the $\mathrm{CoP}$ would converge on a small area located $38 \mathrm{~cm}$ underground (Fig.2). From these models, we have rebuilt the CoM trajectory using the boundary conditions of the single support phase, at TTO and THS.

The resulting trajectories are given in Fig. 3 and compared to the reference trajectory in the frontal, sagittal and horizontal planes, respectively. In Fig. 3, while the left figures isolate the CoM trajectories for a more precise observation, the right ones show a general view including the positions of the pivot points and the boundary rods (TTO and THS) for each model. One can notice wide differences around the vertical axis, as show the sagittal and the frontal views, while the respective positions of the pivot points allow the horizontal trajectories (in the $\mathrm{X}-\mathrm{Y}$ plane) being very similar. Another observation shows that while the reference trajectory is not as circular than the approached ones in the sagittal plane, oppositely it is much more circular in the frontal plane. This is directly related to the fixed length of the pendulum rods in our representations. Indeed, implementing the reference support distance measured between the CoP and the CoM in each models would lead to much more precise approximations: the EIP model would be naturally identical to the reference by definition, while the IP and GIP models would get much closer than when using fixed-length pendulum rods.

Let us focus on the GIP model, more particularly on the convergence area of the support lines joining the $\mathrm{CoP}$ and the CoM. These two points may be considered as representative parameters of the motion dynamics of the subjects. We have observed that the support lines do not cross in the three dimensional space. We have then calculated the minimal distances between two support lines observed at two consecutive time steps, all over the single support phase. Let us define the instantaneous pivot point 
as the middle of these calculated minimal distances, which are represented in Fig. 4 for the complete support phase. In this figure, we can see that the values of the minimal distances are bounded between -0.026 and $+0.27 \mathrm{~mm}$, oriented positively in the direction obtained by the cross product between the two consecutive support lines. We have represented the distribution in space of these minimal distances and their respective orientation in Fig. 5. We have observed for all the walking subjects that the distances are almost null up to mid stance, and become larger after mid stance, following a linear length growth as shown in Fig. 4.

The minimal distances are mainly supported along the lateral axis, and their distribution (length and position in space) with time over the single support phase is illustrated in Fig. 5 using the black lines. Using these data, we have located a mean pivot point of the stance phase by calculating the weighted mean of the minimal distances which is represented in the same Fig. 5 using the red line. The mean distance in very small: $\mathrm{XX} \mathrm{mm}$. The blue line represents the minimal distance between the first and the last support lines of the stance phase (measured at TTO and THS, respectively). We call it the minimal boundary distance as it is based on the boundary support conditions. The distance between the mean pivot point (middle of the mean minimal distance) and the middle of the minimal boundary distance is represented by the green line: this distance remains smaller than $2 \mathrm{~mm}$ (maximum distance observed on the five subjects), which allowed us to consider that taking the boundary conditions of the support lines leaded to a good approximation of the position of the pivot point $P_{0}^{\prime}$. Moreover, comparing the minimal boundary distance and the sum of all the instantaneous minimal distances led to a difference of $0.012 \mathrm{~mm}$ which is negligible.

The evolution of the instantaneous rotation vector between two support lines is shown in Fig. 6. It is mainly oriented along the sagittal axis, its orientation remaining within a conic shape which center is the lateral $Y$-axis. The norm of the rotation vector linearly decreases from $4.8510^{-3}$ to $410^{-3}$ at $60 \%$ of the single support phase, stabilizes until $90 \%$ and suddenly increases up to $4.410^{-3}$. As mentioned previously, its main orientation is along the sagittal axis. Nevertheless, as can be observed in Fig. 6, its projection on the sagittal plane follows a triangular pattern first oriented in the frontal direction, then slowly axing toward the vertical direction and back to the frontal one, then suddenly moving toward an axis of equation $\mathbf{x}-\mathbf{z}$. As for the location of the pivot point, we have compared the weighted mean rotation vector to the average one between TTO and THS. Once again, using an average rotation vector between the bounding support conditions is very similar to the mean average of the rotation vector along the comlplete single support phase.

Finally, the pivot point $P_{0}^{\prime}$ of the GIP-3D model introduced in this paper can be completely defined using the boundary support lines of the single support phase, measured at TTO and LTO respectively. The position of the pivot point is defined by the middle of the smallest distance between these two lines, and the rotation vector results from the cross product between $P_{0}^{\prime} C_{T T O}$ and $P_{0}^{\prime} C_{T H S}$.

\section{CONCLUSION}

We have proposed an inverted pendulum model, denoted $G I P-3 D$, to represent human normal walking. Similarly to other existing models, it is based on the cyclic sinusoidal motion of the center of mass during normal walking. Our model also takes the mechanism of the foot into account by considering that the pivot point of the inverted pendulum is located under the ground level. We have observed more precisely the behavior of the convergence area of the support lines (i.e. the straight lines passing by the CoM and the CoP at each time step) and could determine the position of the pivot point only by considering the support lines at TTO and at LTO. The double support phase is then modeled using an homothetic transformation of the single support phase, which center is the approached CoM $C^{\prime}$ at LTO and ratio $k_{0}$ defined in Eq. 2

This model presents several advantages: similarly to [3], the mechanism of the foot is considered and allows reducing the vertical amplitude of the CoM motion, in comparison to classical approaches which fix the pivot point at ground level and located at the middle of the trajectory described by the CoP. But, in [3]'s approach, the pivot point is not fixed and require precise measurement of the CoP position during the whole stance phase. In our approach, the pivot point position only requires knowing the state of the support lines at the boundary instants of the stance phase, LTO and THS, and is fixed for the whole single support phase. Another important advantage is that this model does not rely on the symmetric characteristics of normal walking, moreover it may be applied to pathological walking as long as the boundary states of each stance phase are known. To realize such applications, the length of the pendulum rod should also be considered as variable with time. The next steps in this study will be to extend and validate the GIP-3D model to pathologic walking using variable-length pendulum rods and continuity equations at the transition instants between the single and the double support phases.

\section{REFERENCES}

[1] George A. Bertos, Dudley S. Childress, and Steven A. Gard. The vertical mechanical impedance of the locomotor system during human walking with applications in rehabilitation. In 9th International Conference on Rehabilitation Robotics, 2005.

[2] Steven A. Gard and Dudley S. Childress. What determines the vertical displacement of the body during normal walking? Journal of Prosthetics and Orthotics, 13(3), pages 64-69, 2001.

[3] Chris Hayot, Sophie Sakka, and Patrick Lacouture. Assessing the respective effects of the gait determinants on the vertical trajectory of the center of mass using the compass gait representation. Gait \& Posture, submitted, pages ., 2010.

[4] Chris Hayot, Sophie Sakka, and Patrick Lacouture. Eip-3d: An extended $3 \mathrm{~d}$ inverted pendulum model to represent normal human walking cycle. Gait \& Posture, submitted, pages ., 2010. 
[5] Kimberlee Jordan, John H. Challis, and Karl M. Newell. Walking speed influences on gait cycle variability. Gait \& Posture, 26(1), pages 128-134, 2007.

[6] Arthur D. Kuo. The six determinants of gait and the inverted pendulum analogy: A dynamic walking perspective. Human Movement Science, 26(4), pages 617-656, 2007.

[7] G. Legnani, C.F. Righettini, and B. Zappa. A homogeneous matrix approach to $3 \mathrm{~d}$ kinematics and dynamics. part 1: theory. Mechanisms and machine Theory, 1, pages 573-587, 1996.

[8] Gregory J. Lelas, Jennifer L.and Merriman, Patrick O. Riley, and D. Casey Kerrigan. Predicting peak kinematic and kinetic parameters from gait speed. Gait \& Posture, 17(2), pages 106-112, 2003.

[9] Tad McGeer. Passive dynamic walking. International Journal of Robotics Research, 9(2), pages $62-82,1990$.

[10] M. Saunders, Verne T. Inman, and Howard D. Eberhart. The major determinants in normal and pathological gait. Journal of Bone and Joint Surgery, 35, pages 543-558, 1953.

[11] G. Stoquart, C. Detrembleur, and T. Lejeune. Effect of speed on kinematic, kinetic, electromyographic and energetic reference values during treadmill walking. Clinical Neurophysiology, 38, pages 105116, 2008.

[12] Ge Wu, Sorin Siegler, Paul Allard, Chris Kirtley, Alberto Leardini, Dieter Rosenbaum, Mike Whittle, Darryl D. D'Lima, Luca Cristofolini, Hartmut Witte, Oskar Schmid, and Ian A.F. Stokes. Isb recommendation on definitions of joint coordinate system of various joints for the reporting of human joint motion - part i: ankle, hip, and spine. Journal of Biomechanics, 35, pages 543-548, 2002.

[13] Ge Wu, Frans C.T. van der Helm, H.E.J. Veeger, Mohsen Makhsous, Peter Van Roy, Carolyn Anglin, Jochem Nagels, Andrew R. Karduna, Kevin McQuade, Xuguang Wang, Frederick W. Werner, and Bryan Buchholz. Isb recommendation on definitions of joint coordinate systems of various joints for the reporting of human joint motion part ii: shoulder, elbow, wrist and hand. Journal of Biomechanics, 38(5), pages 981-992, 2005.

[14] V.M. Zatsiorsky. Kinetics of Human Motion. The Pennsylvania State University, 2002.

[15] Wiebren Zijlstra and At L. Hof. Displacement of the pelvis during human walking: experimental data and model predictions. Gait \& Posture, 6(3), pages 249-262, 1997. 